\title{
Title: Advancing Research Using Action Cameras
}

Authors: Mattias Björnmalm, Matthew Faria, and Frank Caruso*

Affiliation: ARC Centre of Excellence in Convergent Bio-Nano Science and Technology, and the Department of Chemical and Biomolecular Engineering, The University of Melbourne, Parkville, Victoria 3010, Australia.*E-mail: fcaruso@unimelb.edu.au

Main Text: Many of today's most pressing challenges require multidisciplinary and multinational collaboration. For example, to meet the needs of tomorrow's healthcare we are moving toward convergence of the physical sciences, engineering, and biomedicine. ${ }^{1,2}$ Central to this development is making it easier to understand the diverse experiments, techniques and methods that exist in disparate but increasingly connected fields. This includes making experimental sections as clear and easy to follow as possible, ${ }^{3}$ and making use of reporting practices, ${ }^{4}$ both of which are important parts of any open research culture promoting transparency, openness, and reproducibility. ${ }^{5}$ However, communicating important but subtle details in procedures and processing can be difficult, especially to an interdisciplinary audience. For example, the US National Institutes of Health has identified insufficient protocol description as a key contributor to current challenges associated with reproducibility. ${ }^{6}$ Imagine how much easier this process would be if we could describe parts of our research as we are performing it to someone who is seeing exactly what we are seeing?

We have recently explored the use of first-person action cameras (e.g., GoPro cameras) to record experiments, methods, and equipment procedures in our lab. ${ }^{7}$ We are also recording the training sessions for our equipment and instruments, our lab inductions, and (bio)safety procedures to complement our conventional training. Our intention is to facilitate transfer of knowledge vertically and horizontally, internally and externally. For example, internally many labs (including our own) have to manage the challenge of transferring knowledge when senior group members move on and new colleagues join. Externally, there are collaborators and colleagues all over the world working with similar problems using 
similar tools. Using small, hands-free action cameras (worn like headlamps), researchers can easily explain and demonstrate their research (and the equipment and methods they are experts on) from their own perspective as they are doing it (Figure 1). These cameras have become widely available and it is easier and cheaper than ever to share videos; thus we believe there is great potential for researchers around the world to use recordings to share their specific expertise and experience. This could not only accelerate and increase the impact of their own research, but also advance interdisciplinary research endeavors and other fields.

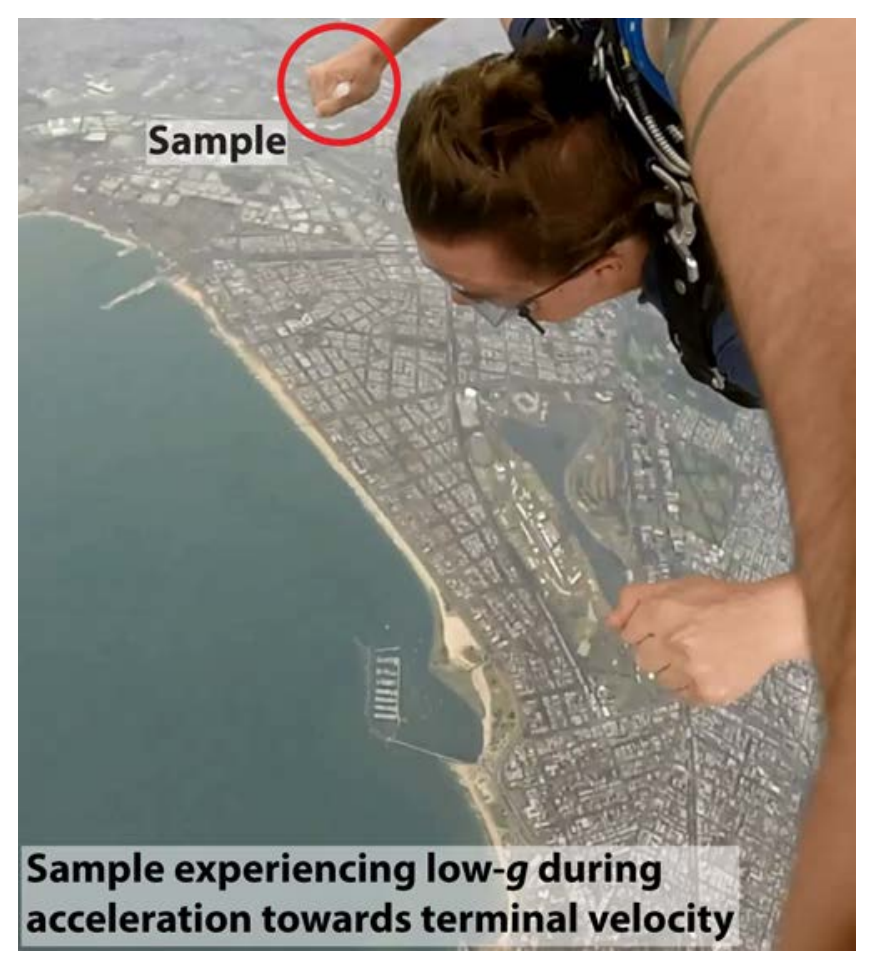

Figure 1. Snapshot of a video showing an experimental procedure involving simulated low gravity. ${ }^{8}$

\section{References}

(1) Björnmalm, M.; Faria, M.; Caruso, F. Increasing the Impact of Materials in and beyond Bio-Nano Science. J. Am. Chem. Soc. 2016, 138, 13449-13456.

(2) Sharp, P.; Jacks, T.; Hockfield, S. Capitalizing on Convergence for Health Care. Science 2016, 352, 1522-1523.

(3) Buriak, J. M.; Korgel, B. The Experimental Section: The Key to Longevity of Your Research. Chem. Mater. 2014, 26, 1765-1766.

(4) Buriak, J. M.; Jones, C. W.; Kamat, P. V.; Schanze, K. S.; Schatz, G. C.; Scholes, G. D.; Weiss, P. S. Virtual Issue on Best Practices for Reporting the Properties of Materials and Devices. Chem. 
Mater. 2016, 28, 3525-3526.

(5) Nosek, B. A.; Alter, G.; Banks, G. C.; Borsboom, D.; Bowman, S. D.; Breckler, S. J.; Buck, S.; Chambers, C. D.; Chin, G.; Christensen, G.; Contestabile, M.; Dafoe, A.; Eich, E.; Freese, J.; Glennerster, R.; Goroff, D.; Green, D. P.; Hesse, B.; Humphreys, M.; Ishiyama, J.; Karlan, D.; Kraut, A.; Lupia, A.; Mabry, P.; Madon, T.; Malhotra, N.; Mayo-Wilson, E.; McNutt, M.; Miguel, E.; Paluck, E. L.; Simonsohn, U.; Soderberg, C.; Spellman, B. A.; Turitto, J.; VandenBos, G.; Vazire, S.; Wagenmakers, E. J.; Wilson, R.; Yarkoni, T. Promoting an Open Research Culture. Science 2015, 348, 1422-1425.

(6) Collins, F. S.; Tabak, L. A. NIH Plans to Enhance Reproducibility. Nature 2014, 505, 612-613.

(7) Björnmalm, M.; Cui, J.; Bertleff-Zieschang, N.; Song, D.; Faria, M.; Rahim, M. A.; Caruso, F. Nanoengineering Particles through Template Assembly. Chem. Mater. 2016, DOI: 10.1021/acs.chemmater.6b02848.

(8) Richardson, J. J.; Liang, K.; Lisi, F.; Björnmalm, M.; Faria, M.; Guo, J.; Falcaro, P. Controlling the Growth of Metal-Organic Frameworks Using Different Gravitational Forces. Eur. J. Inorg. Chem. 2016, 2016, 4499-4504. 


\section{University Library}

\section{- M M I N E R VA A gateway to Melbourne's research publications}

Minerva Access is the Institutional Repository of The University of Melbourne

Author/s:

Bjornmalm, M;Faria, M;Caruso, F

Title:

Advancing Research Using Action Cameras

Date:

2016-12-13

Citation:

Bjornmalm, M., Faria, M. \& Caruso, F. (2016). Advancing Research Using Action

Cameras. CHEMISTRY OF MATERIALS, 28 (23), pp.8441-8442. https://doi.org/10.1021/ acs.chemmater.6b04639.

Persistent Link:

http://hdl.handle.net/11343/123237 